\title{
Wahrheit und Wirklichkeit
}

\section{Tagungsbericht}

\section{Andreas Hunziker}

"Das ist aber wirklich wahr! - schon dieser alltägliche Ausruf zeigt, wie eng die beiden Begriffe ,Wahrheit und >Wirklichkeit zusammenhängen. Wenn darum die dreiteilige - vom Institut für Hermeneutik und Religionsphilosophie organisierte - Tagungsreihe zur Wahrheitsproblematik am 21./22. Juni 2002 mit sechs Vorträgen zum Thema "Wahrheit und Wirklichkeit" abgeschlossen wurde, schien damit auch so etwas wie der entscheidende Punkt in Sachen ,Wahrheit erreicht worden zu sein: die Wirklichkeit. Nur, die Wirklichkeiten, in denen wir leben, sind so vielfältig wie unser Leben selber, so dass von 'wirklich Verschiedenem und in vielen unterschiedlichen Kontexten Gebrauch gemacht wird. Dies bestätigte sich m.E. auch in denjenigen Vorträgen dieser Tagung, die philosophisch eine emphatischere Variante des »Das ist aber wirklich wahr! anstrebten. Intensivere Diskussionen hat es dabei insbesondere darüber gegeben, ob sprachphilosophisch und wahrheitstheoretisch der Semantik oder der Pragmatik der Primat gebührt (Puntel vs. Rust) und über die damit zusammenhängende Problematik, wie Glaubensleben zum einen und philosophische sowie theologische Rede von Gott und Wahrheit zum andern aufeinander zu beziehen sind (Herrmann, Kreiner).

Der gerade von seinen Publikationen zum Wahrheitsthema bekannte Philosoph L.B. Puntel versuchte "Wahrheit als semantischontologischen Grundbegriff $z$ zu entfalten. Ausgehend von der sprachphilosophischen Doppelthese der Fundamentalität der semantischen Dimension der Sprache und der Angewiesenheit der sprachlichen Bedeutung auf Volldetermination, explizierte er den ontologischen Bezug von $/ W$ ahr(heit)< als Identität zwischen einer wahren Proposition und einer Tatsache. Ohne die mit der traditionellen Korrespondenztheorie verbundenen Schwierigkeiten zu übernehmen, wird damit Identität als sperfekter Fall von Korrespondenz verstanden, so dass der unaufgebbare Kern der Korrespondenztheorie erhalten bleibe: der Bezug zur Welt als absolut wesentliches Ingrediens von Wahrheit. 
Der Beitrag des Cambridgers D.H. Mellor - »a metaphysical realist well able to counter the subjectivist and relativist leanings of many other leading philosophers of science« (The Oxford Companion to Philosophy, 1995) - war auf eine Verteidigung des »Truthmaker«-Begriffs aus. Mellor ging so vor, dass er die Reichweite des Wahrmacher-Begriffs begrenzte, um so unnötige Einwände gegen diesen ins Leere laufen zu lassen. Wenn dabei nach Mellor die Vorstellung des Wahrmachens von der Vorstellung herstammt, Wahrheit sei Korrespondenz mit der Wirklichkeit, ohne damit aber eine Korrespondenztheorie der Wahrheit oder eine bestimmte Bedeutungstheorie $\mathrm{zu}$ implizieren, spiegelte sich hier eine ähnliche Problemlage wie bei Puntel, auf die Mellor aber anders reagiert: Mit wissenschaftlichen und metaphysischen Theorien darüber, welche Wahrmacher es gibt und was sie wahr machen einerseits, und mit einer philosophischen Wahrmachertheorie andererseits, die zum einen fragt, welche Propositionen überhaupt Wahrmacher benötigen und die uns zum andern daran erinnert, dass der Semantik die Metaphysik zugrunde liegt, und nicht umgekehrt.

Der Zürcher Philosoph A. Rust zeigte in seinem Vortrag »Wo Wahrheit zum Problem wird«, dass Wahrheit dort zum Problem wird, wo wir uns über den implizit-alltäglichen Begriff der Wahrheit in Richtung einer philosophischen Theorie der Wahrheit hinauszubewegen versuchen. Davon zeuge nicht nur die Korrespondenztheorie der Wahrheit, sondern auch die oft als Alternative dazu angesehene Kohärenztheorie, die aber ähnlich problematisch wie erstere sei. Rust unterschied sich vor allem von Puntel darin, dass er zwar an der alten Common-sense-Bestimmung der Wahrheit als Übereinstimmung von Aussage und Sachverhalt festhalten wollte, die Probleme aber dort lozierte, wo dieses intuitive Wahrheitsverständnis in problematischer Weise zu präzisieren versucht wird.

Der Theologe J. Rohls aus München bot in seinem Vortrag "Denken, Sein und Wahrheit» einen eher geschichtlich ausgerichteten Überblick über verschiedene Wahrheitsmodelle. Wobei er voraussetzte, dass sich diese alle als Bearbeitungen derselben Problematik und darum als einen großen Diskussionszusammenhang verstehen lassen: das pragmatistische Wahrheitsverständnis (Rorty), die Korrespondenztheorie der Wahrheit (Russel, früher Wittgenstein), die Konsenstheorie der Wahrheit (Peirce) und die Kohärenz- 
theorie der Wahrheit (Davidson). Abgeschlossen wurde dieser Vortrag durch einige »theologische Randbemerkungen«.

In seinem Vortrag "Wir Menschen, Wahrheit und Wirklichkeit« erläuterte der Philosoph Eberhard Herrmann aus Uppsala zuerst, was unter Wahrheit als konstituierter Wirklichkeit zu verstehen ist und mit welcher Art von Relativismus diese Auffassung verbunden ist; dann kritisierte er den nicht-epistemischen Wahrheitsbegriff des metaphysischen Realismus, um statt dessen einen normativen Wahrheitsbegriff in epistemischer Perspektive zu entwickeln. Schließlich versuchte er zu zeigen, wie in dazu analoger Weise von Wahrheit in existenzieller Perspektive die Rede sein kann: In Bezug auf die Frage, was es bedeutet, Mensch zu sein, das heißt, mit den Unumgänglichkeiten des Lebens zu leben, bedeutet >wahr $>$ in einer hierfür geeigneten materialen Definition sübereinstimmend mit der von uns konzeptualisierten Wirklichkeit, wie sie uns in unseren Erfahrungen Widerstand leistet den, welche Aussagen wahr und welche falsch sind, sind hier Kohärenz und existenzielle Adäquanz.

Einen in doppelter Hinsicht anderen Zugang als seine Vorredner wählte der Theologe A. Kreiner aus Mainz mit seinem Referat "Wahrheit und Perspektivität religiöser Rede von Gott«. Nicht nur war seine Argumentation explizit theologischer Natur. Sondern auch seine Behauptung, dass Aussagen über Gott, wenn sie wahr sind, dann in gleichem (korrespondenztheoretischen) Sinne wahr sein müssten, wie Aussagen über andere Dinge, gab seinem Vortrag sein eigentümliches Profil. In einem ersten Schritt versuchte Kreiner, die These zu widerlegen, dass über Gott nur sagbar sei, was er nicht sei. Dann setzte er sich mit dem s.M.n. gravierendsten Problem der Rede von Gott auseinander, nämlich der äußerst unterschiedlichen Verwendung des Begriffs `Gott $८$. Kreiner suchte darum nach - nicht nur traditionsinternen - rationalen Kriterien für die Rede von Gott, wobei über die nicht hinreichenden logischen, wirklichkeitserschließenden und pragmatischen Kriterien die an Anselm anknüpfende Regel entscheidend wird, dass von Gott nur das aussagbar sei, was ihn als das für uns Wichtigste und Vollkommenste verstehbar macht. Da dieses Kriterium aber abhängig von unseren intuitiven Werten sei, sei mit ihnen auch der Gottesbegriff ständiger Veränderung unterworfen. Folgerichtig schloss der Vortrag mit Kreiners eigener Wertintuition, dass >Person 
das Wertvollste bezeichne, was das Universum bis heute hervorgebracht habe. Gott sei darum personhaft - mit Bewusstsein, Macht und Wille - zu denken.

Die Vorträge und Diskussionen machten noch einmal deutlich, womit diese Tagungsreihe ein Jahr vorher begonnen hatte: Wahrheit gibt es nur in Horizonten, Horizonte zudem die individuell sehr verschieden sein können. Und an diesem Ort, unseren nicht auf den Begriff zu bringenden Horizonten, zeigte sich dann noch einmal anschaulich, was Thema der vorangegangen Tagung gewesen war: wie eng eben auch Wahrheit und Rhetorik zusammengehören. Vielleicht weil beim Wahrheitsthema unser Bedürfnis besonders stark zum Audruck kommt, den andern davon zu überzeugen, dass mein Verständnis davon »aber wirklich wahr ist $\lll$.

Andreas Hunziker (VDM) ist Assistent am Lehrstuhl für Systematische Theologie bei Prof. I.U. Dalferth. 